\title{
International Journal of Electronics and Electrical Engineering
}

\section{CONTENTS}

\section{Volume 1, Number 1, March 2013}

Reducing Massage Passing and Time Complexity in Bully Election Algorithms Using Two Successors 1 Basim Alhadidi, Laith H. Baniata, Mohammad H.Baniata, and Mohammad Al-Sharaiah

On the Design and Implementation of an Efficient Information Retrieval System for Arabic Language 5 Mohammad O. Wedyan and Aarti Singh

Bilateral Filter Based Selective Unsharp Masking Using Intensity and/or Saturation Components. 10 Noriaki Suetake, Yohei Kuramoto, Eiji Uchino, Kazuhiro Tokunaga, and Sadanori Hirose

Framework for Transaction Execution Strategies in Mobile Data Base Systems

Ahmad al-Qerem

Managing E-Waste Using TRIZ

Nikalus Shu Luing Swee, Mum Wai Yip, Chee Sheng Keong, See Chew Tai, and Guat Guan Toh

Effect of High Performance SiGe HBT Design Parameters on the Minimum Gate Delay of an ECL Inverter .23 Chafia Yahiaoui

A Power and Area Efficient 8-Channel Neural Signal Front End for Biomedical Applications .26

Sami ur Rehman and Awais Mehmood Kamboh

Comparison of Total Ionizing Dose Effects for Floating and Tied Body SOI nMOSFETs .31

Bingxu. Ning and Zhengxuan. Zhang

A Reliable, Process-Sensitive-Tolerant Hybrid Sense Amplifier for Ultralow Power SRAM 34

B.S. Reniwal and S. K. Vishvakarma

Architectural Evaluation of Asymmetric Algorithms in ARM Processors

Gustavo S. Quirino and Edward David Moreno

A 6.5- $\mu \mathrm{V} /{ }^{\circ} \mathrm{C}$ Offset Drift Compensation Technique for Dynamic Comparator

Daiki Tabira and Kenichi Ohhata

A Low-Power 16-bit RISC Microcontroller

Li Guangcai, Lu Tiejun, and Zong Yu

CCTAs based Current-mode Quadrature Oscillator with High Output Impedances

T. Thosdeekoraphat, S. Summart, C. Saetiaw, S. Santalunai, and C. Thongsopa

A Hybrid Intelligent Autonomous Model Developed Using Multi-Agent Systems.

Anusua Ghosh, Andrew Nafalski, and Jeffery W Tweedale 